\title{
Activation spectroscopy of electronically induced defects in solid $\mathrm{Ne}$
}

\author{
O.N. Grigorashchenko ${ }^{1}$, V.V. Rudenkov ${ }^{1}$, I.V. Khizhnyi ${ }^{1,2}$, E.V. Savchenko ${ }^{1}$, \\ M. Frankowski ${ }^{3,4}$, A.M. Smith-Gicklhorn ${ }^{3}$, M.K. Beyer ${ }^{3}$, and V.E. Bondybey ${ }^{3}$ \\ ${ }^{1}$ B. Verkin Institute for Low Temperature Physics and Engineering of the National Academy of \\ Sciences of Ukraine, 47 Lenin Ave., Kharkov 61103, Ukraine
}

E-mail: savchenko@ilt.kharkov.ua

${ }^{2}$ V. Karazin Kharkov National University, 4 Svobody Sq., Kharkov 61107, Ukraine

3 Institute of Physical and Theoretical Chemistry, TU Munich, Garching 85747, Germany

E-mail: bondybey@ch.tum.de

${ }^{4}$ Institute of Fluid-Flow Machinery, Polish Academy of Sciences, 14 J. Fiszera, Gdansk 80-231, Poland

Thermally stimulated luminescence (TSL) and thermally stimulated exoelectron emission (TSEE) methods were used in combination with cathodoluminescence to probe electronically induced defects in solid Ne. The defects were generated by a low energy electron beam. For spectroscopic study we used $\mathrm{Ar}^{*}$ centers in Ne matrix as a model system. At a temperature of $10.5 \mathrm{~K}$ a sharp decrease in the intensity of «defect» components in the luminescence spectrum was observed. From the analysis of the corresponding peak in the TSL and TSEE yields the trap depth energy was estimated and compared with available theoretical calculations. The obtained data support the model suggested by Song, that stable electronically induced defects have the configuration of second-neighbour Frenkel pairs.

PACS: 78.60.Kn, 79.75.+g

\section{Introduction}

Activation spectroscopy is a powerful tool for the study of defects in solids [1]. Electronically induced defects represent a special class of lattice defects created via an electronic subsystem. The basis for the physics of their formation is a concentration of the electronic excitation energy within a volume about that of a unit cell followed by the energy release. These defects were actively studied on a variety of materials [2-4]. Atomic cryocrystals with their simple lattice and well-known electronic structure are especially suitable for these studies due to small binding energies in conjunction with a strong exciton-phonon interaction. Electronically induced stable lattice defects were found in solid $\mathrm{Ne}, \mathrm{Ar}, \mathrm{Kr}$, and Xe [5] using spectroscopic methods in experiments carried out with a low energy electron beam. It was suggested that the stimulating factor is the self-trapping of excitons either into molecular-type self-trapped excitons (M-STE) or atomic-type (A-STE) states. In solid Ne the main channel of the exciton self-trapping is the self-trapping into A-STE states. The recent state-selective study performed with synchrotron radiation in the range of excitonic absorption $n=2 \Gamma(3 / 2)$ gave a direct evidence of the formation and accumulation of point lattice defects via the excitonic mechanism [6]. The excited state molecular dynamics study of the possible evolution of the self-trapped excitons into stable lattice defects has shown that the self-trapping may result in Frenkel pair creation, and a microscopic structure of the defects has been proposed [7]. However, to our best knowledge so far no experimental study of electronically induced defects structure in solid Ne exists.

We applied activation spectroscopy methods to get information on the charge traps related to electronically induced permanent lattice defects in solid Ne. The most common method in use is thermally stimulated luminescence (TSL) [1]. However, an interpretation of TSL data is hampered by the fact that the emission of light may be caused by recombination of 
both charged species and neutral ones. In these cases the meaning of activation energies derived from experimental data could be quite different. In order to distinguish between the recombination processes of neutral and charged species, TSL is combined with methods of current activation spectroscopy, as demonstrated in recent experiments performed with solid Ar $[8,9]$. Thermally stimulated currents (TSC) were measured along with TSL by Schrimpf et al. [8]. The method of thermally stimulated exoelectron emission (TSEE) enabled us to prove directly that the electrons are the mobile charge carriers responsible for recombination reactions in irradiated rare gas solids [9]. In this study we also used the TSEE method in combination with TSL to probe electronically induced defects in solid $\mathrm{Ne}$.

\section{Experimental section}

A detailed description of the experimental procedure is given elsewhere [9-11]. High-purity Ne $(99.999 \%)$ gas was used. The samples were grown from the gas phase by pulsed deposition on a substrate cooled by a closed-cycle 2-stage Leybold RGD 580 cryostat to a temperature of $6 \mathrm{~K}$. The metal substrate was coated with a thin layer of $\mathrm{MgF}_{2}$. The gas-handling system was annealed and pumped before the experiments. The pressure in the sample chamber was $6 \cdot 10^{-8}$ mbar. Samples were deposited with a concurrent irradiation by electrons with an energy of $150 \mathrm{eV}$, which is insufficient for producing point defects via the knock-on mechanism. A typical deposition rate was $10^{-2} \mu \mathrm{m} \cdot \mathrm{s}^{-1}$. We deposited Ne films of $50-100 \mu \mathrm{m}$ thickness. The sample thickness and the deposition rate were determined by measuring the pressure decrease in a known volume of the Ne reservoir in the gas-handling system. The samples had a high optical quality and looked transparent in the visible range.

Defect levels in the energy gap were probed by measuring thermally stimulated exoelectron emission and thermally stimulated luminescence. The programmable temperature controller permitted to keep the desired temperature during sample preparation and irradiation, as well as to control the heating rate. The temperature was measured with a calibrated silicon diode sensor, mounted at the substrate. In the TSEE and TSL experiments samples were heated with a constant rate of $1.6 \mathrm{~K} \cdot \mathrm{min}^{-1}$. The measurements were performed in the temperature range of 6-12 K. The emission of electrons from preirradiated samples was detected with a movable Au-coated Faraday plate kept at a small positive potential of $+9 \mathrm{~V}$. During measurements it was positioned at a distance of $5 \mathrm{~mm}$ in front of the sample, which had been grown on a grounded substrate. The current from the Faraday plate was am- plified by a FEMTO DLPCA 100 current amplifier. The signal was reversed in polarity and digitized in a PC. The total yield of TSL was detected with a photomultiplier tube (PMT). By introducing a converter of vacuum ultraviolet radiation into visible light we were able to extend the available spectral range and detect TSL from $800 \mathrm{~nm}$ to $10 \mathrm{~nm}$.

In addition luminescence spectra from nominally pure and Ar doped solid Ne were detected in vacuum ultraviolet (VUV) range under excitation by low energy electrons. The dose dependence and the influence of heating on the luminescence intensity and spectral distribution were examined.

\section{Results and discussion}

In a perfect lattice of solid Ne electrons are delocalized, and their transport exhibits free-electron behavior as it has been clearly indicated in experiments using time-of-flight [12] and muon spin rotation/relaxation $\left(\mu^{+} \mathrm{SR}\right)$ [13] techniques. The second important point is a large mean free path of the conduction electrons in cryocrystals of high structural quality [14]. It makes it possible to use the TSEE method of current activation spectroscopy for traplevel studies, and one can expect to get information on the volume traps formed within the sample under irradiation. In view of the negative electron affinity $E_{a}=$ $=-1.3 \mathrm{eV}$ of solid $\mathrm{Ne}$ [2] only such kinds of structural defects like vacancies, vacancy clusters or pores may serve as efficient shallow traps for electrons. In addition, traces of impurities, which can be considered as «electron scavengers» $\left(\mathrm{O}_{2}, \mathrm{O}, \mathrm{OH}\right.$, etc. $)$, form deep, thermally disconnected electron traps. The electrons from these traps can only be released optically.

At low temperature, after the irradiation was stopped, we observed a long «afterglow» of luminescence and «afteremission» of electrons from $\mathrm{Ne}$ samples. The phosphorescence is due to minor traces of impurities. Such an afterglow was observed very often in matrix isolation studies $[15,16]$. The «afteremission» of electrons is supposed to be stimulated by afterglow photons similarly to the effect observed in nitrogen doped solid Ar [17]. TSL and TSEE yields were detected when «afteremission» and afterglow were completed. During annealing, if there is enough energy to release the electrons from the traps, they can either recombine with positively charged centers with subsequent emission of light or they can be detected as a thermally stimulated emission of electrons.

The typical yields of TSL photons and TSEE currents taken from Ne samples grown under the electron beam are shown in Fig. 1. We have found a pronounced maximum in the TSEE and TSL yields at $10.5 \mathrm{~K}$. In both cases the peak is not isolated - the 


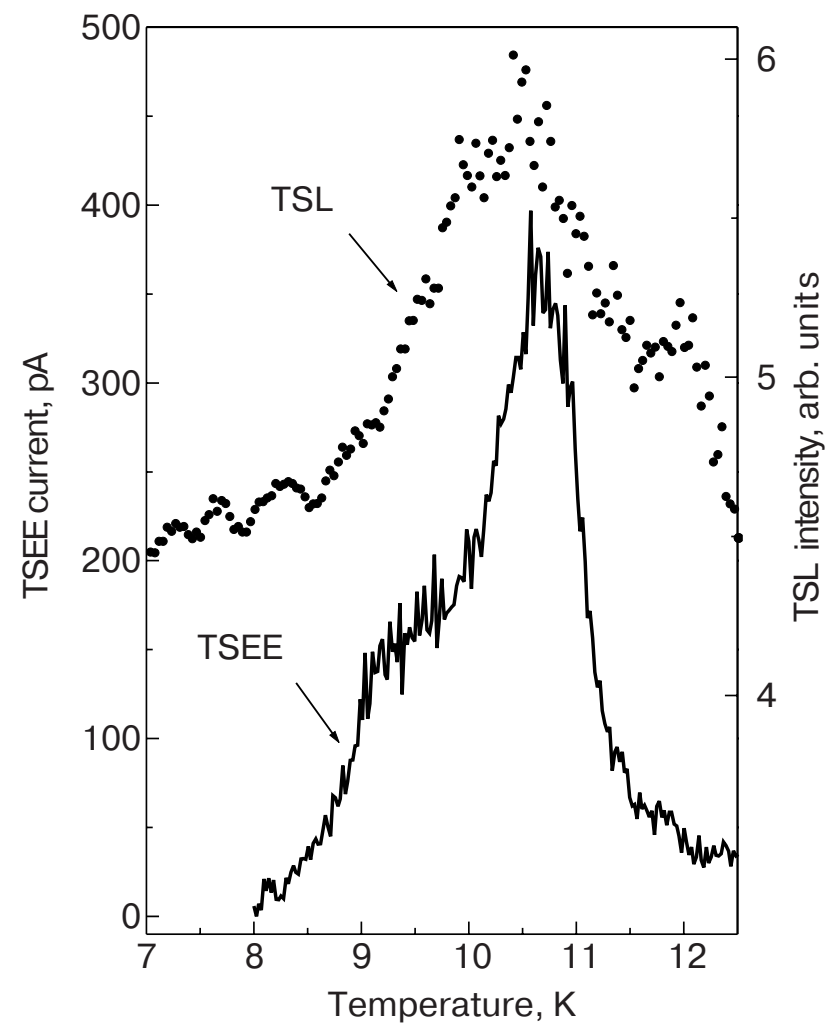

Fig. 1. Integrated yield of TSL and yield of TSEE taken from solid Ne.

«current curve» and especially the «glow curve» exhibit overlapping peaks. Note, that the TSEE curve has a more simple structure. Apart from the main peak at $10.5 \mathrm{~K}$ a wide low temperature shoulder was seen around $9 \mathrm{~K}$. No intensive peaks at the higher temperature side of the TSEE curve were detected. First of all note the correlation in the position of the main «thermopeak» at $10.5 \mathrm{~K}$ in the yields of both photons and electrons. The observation of TSL and TSEE peak at the same temperature is a clear indication of the common origin of recombined and emitted electrons. The absence of any shift in the peak position between TSL and TSEE is due to the negative electron affinity of solid Ne, i.e. electrons have no barrier to escape the sample. Moreover, a conduction electron will experience an increase of kinetic energy when it exits the surface.

To get information on the trap level related to electronically induced defects one should analyse the peak at $10.5 \mathrm{~K}$, which exhibits characteristic dose behavior. One more reason to consider this peak as related to electronically induced defects comes from the experiments on Ar doped Ne matrix. It was shown theoretically [7] and tested experimentally [5] that the processes of structural defect creation in the system Ar atom in Ne lattice (model of A-STE center) are quite similar to those in pure solid Ne. In other words an $\mathrm{Ar}$ atom could be used as a «probe» to test the processes in Ne matrix. This system is more convenient for spectroscopic study because of the sharper structure of the luminescence band. The lattice expands around the excited $\mathrm{Ar}^{*}$ center in response to the repulsive interaction of the excited electron and the surrounding atoms of the Ne matrix. After the formation of the primary bubble some of the $\mathrm{Ne}$ atoms from the surrounding could be removed from the first solvation shell and fitted into second or third shell, overcoming the barrier which leads to the defect position. It was found that the second-nearest neighbor vacancy-interstitial pairs could evolve into lattice defects, which remain stable after the electronic transition of the $\mathrm{Ar}^{*}$ center into the ground state. The estimated difference in the energy of the electronic transitions in the primary bubble around $\mathrm{Ar}^{*}$ center and the bubble associated with the defect is reported as $105 \mathrm{meV}$ [7]. The luminescence of $\mathrm{Ar}^{*}$ centers in solid Ne in the range of the electronic transitions from ${ }^{1} P_{1}$ and ${ }^{3} P_{1}$ states was studied previously [18], and a sharp intensity redistribution was found at $10.5 \mathrm{~K}$. In connection with our current study we reinvestigated the luminescence of $\mathrm{Ar}^{*}$ centers in Ne matrix and its temperature behavior. The experiments were carried out with samples of better structural quality than before [18]. The luminescence spectrum shown in Fig. 2 consists of two bands $\langle a \gg$ and $\langle b »$ of doublet structure. The $a$ - and $b$-bands were identified as ${ }^{1} P_{1} \rightarrow{ }^{1} S_{0}$ and ${ }^{3} P_{1} \rightarrow{ }^{1} S_{0}$ transitions [18], respectively. The high-energy component $\ll 2 »$ belongs to the emission from the primary bubble, the low-energy component «1» is related to the stable electronically induced lattice defects as it was found before [5] by measuring the dose dependence. The luminescence band difference between «1» and «2» components in the spectrum is about $100 \mathrm{meV}$, in good agreement with theoretical data [7]. Upon heating the samples we observed some increase in the relative intensity of the defect related component «1» for both transitions. As the temperature reached $10.5 \mathrm{~K}$ the intensity of defect components related to the electronically induced defects decreased drastically. A subsequent cycle of irradiation at low temperature restored the intensity distribution in the luminescence spectrum of the $\mathrm{Ar}^{*}$ center. The temperature dependence of the relative intensities of the components for the singlet-singlet and the triplet-singlet transitions is shown in Fig. 2. The observed intensity redistribution could be interpreted as annealing of the electronically induced defects. Note that sharp changes in the luminescence spectra of solid $\mathrm{Ne}$ at $10.5 \mathrm{~K}$ were detected also in experiments with $\mathrm{x}$-ray irradiation [19]. 


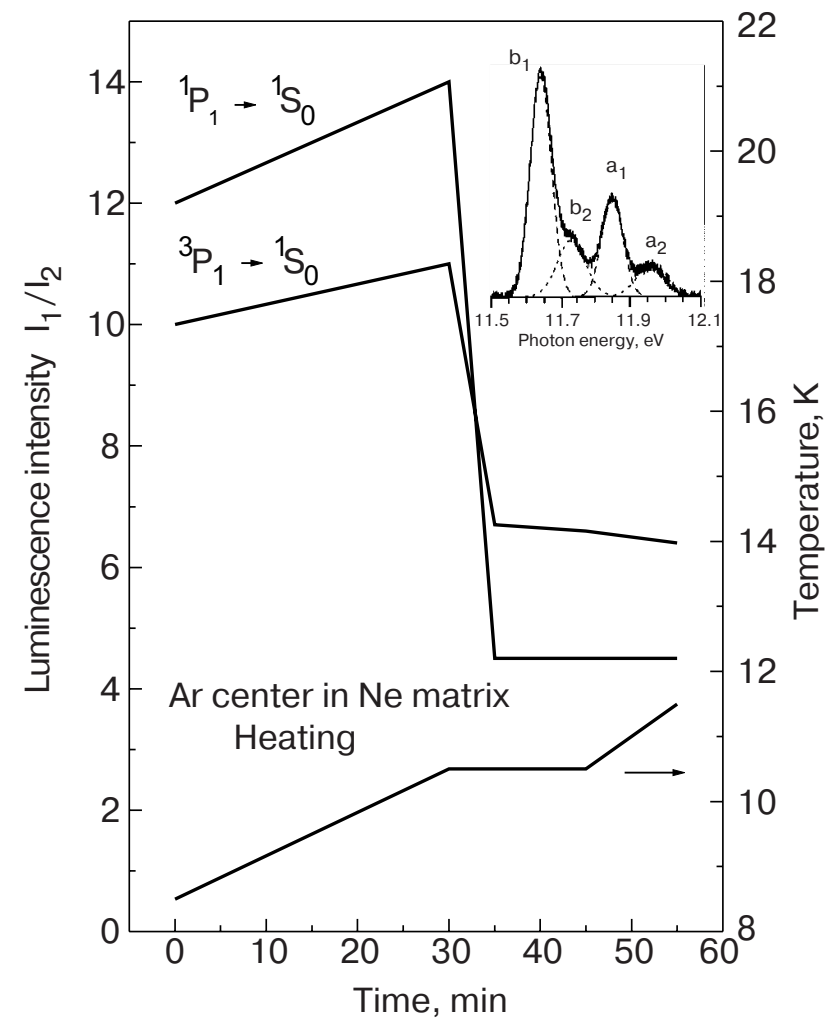

Fig. 2. Luminescence spectrum of $\mathrm{Ar}^{*}$ center in Ne matrix detected under irradiation by low-energy electrons (inset). The $a$-band corresponds to the ${ }^{1} P_{1} \rightarrow{ }^{1} \mathrm{~S}_{0}$ transition, the $b$-band - to the transition from the ${ }^{3} P_{1}$ state. The temperature dependence of the relative intensities of the defect $(\ll 1 »)$ and regular $(\ll 2 »)$ components is plotted for both transitions. The variation of the temperature with time is shown by the curve at the bottom.

Because the peak at $10.5 \mathrm{~K}$ in the TSL curve taken from solid $\mathrm{Ne}$ is strongly influenced by overlapping peaks, we used the TSEE curve for the analysis. Taking into account that the high-temperature part of the TSEE curve is practically free from overlapping, we used for the estimation of the trap depth energy $E_{t}$ the method suggested by Lushchik [20], where only the descending part of a peak in glow curve is used. This method is a version of the half-width method [1]. It was supposed that the experimental data could be fitted using first-order kinetics under the condition of no retrapping. In this case

$$
E_{t}=k T_{m}^{2} /\left(T_{2}-T_{m}\right),
$$

where $T_{m}$ is the temperature at the maximum of the peak and $T_{2}$ is the temperature on the high-temperature side of the peak corresponding to half the maximum intensity. $E_{t}$ estimated by this method from the TSEE experiment is $20 \mathrm{meV}$. This value is in good agreement with the barrier height calculated by Song [7] for the formation of the second neighbor Frenkel pairs - the stable configuration of the electronically induced defects. This barrier prevents vacancy-interstitial pairs from annihilation at low temperatures. Upon annealing the traps disappear, and we observe emission of the electrons. The agreement of the activation energy of the intrinsic electron traps reported here with the energy of thermally activated recombination of the Frenkel pairs seems to be a strong argument in favor of the configuration of stable electronically induced defects suggested previously [7]. Note, that the vacancies in the Frenkel pairs serve as the electron traps.

\section{Acknowledgements}

We thank Profs. K.S. Song and G. Zimmerer for valuable discussions. Financial support from the Deutsche Forschungsgemeinschaft through the program «Förderung der wissenschaftlichen Beziehungen deutscher Wissenschaftler zu Wissenschaftlern in Ländern Mittel- und Osteuropas sowie Ländern der vormaligen UdSSR» is gratefully acknowledged.

1. D.R. Vij, in: Luminescence of Solids, D.R.Vij (ed.), Plenum Press, New York (1998).

2. K.S. Song and R.T. Williams, Self-Trapped Excitons, Springer Series in Solid State Science, Vol. 105, Springer-Verlag, Berlin (1996).

3. M.A. Elango, Elementary Inelastic Radiation-Induced Processes, American Institute of Physics, New York (1991).

4. N. Itoh and A.M. Stoneham, Radiat. Eff. Def. Solids 155, 277 (2001).

5. E.V. Savchenko, A.N. Ogurtsov, and O.N. Grigorashchenko, Phys. Solid State 40, 831 (1998).

6. E.V. Savchenko, A.N. Ogurtsov, and G. Zimmerer, Fiz. Nizk. Temp. 29, 356 (2003) [Low Temp. Phys. 29, 270 (2003)].

7. Chun-rong $\mathrm{Fu}$ and K.S. Song, J. Phys.: Condens. Matter 9, 9785 (1997).

8. A. Schrimpf, C. Boekstiegel, H-J. Stöckman, T. Bornemann, K. Ibbeken, J. Kraft, and B. Herkert, J. Phys.: Condens. Matter 8, 3677 (1996).

9. E.V. Savchenko, O.N. Grigorashchenko, A.N. Ogurtsov, V.V. Rudenkov, G.B. Gumenchuk, M. Lorenz, A. Lammers, and V.E. Bondybey, J. Low Temp. Phys. 122, 379 (2001).

10. E.V. Savchenko, O.N. Grigorashchenko, A.N. Ogurtsov, V.V. Rudenkov, G.B. Gumenchuk, M. Lorenz, M. Frankowski, A.M. Smith-Gicklhorn, and V.E. Bondybey, Surf. Sci. 507-510, 753 (2002).

11. E.V. Savchenko, O.N. Grigorashchenko, A.N. Ogurtsov, V.V. Rudenkov, G.B. Gumenchuk, M. Lorenz, A.M. Smith-Gicklhorn, M. Frankowski, and V.E. Bondybey, Surf. Rev. Lett. 9, 353 (2002).

12. W.E. Spear and P.G. Le Comber, Electronic Transport Properties, in: Rare Gas Solids, M.L. Klein and 
J.A. Venables (eds.), Academic press, New York, (1977), p.1120 .

13. V.G. Storchak, D.G. Eshchenko, J.H. Brewer, S.P. Cottrell, S.F.G. Cox, Karlsson, and R.W. Wappling, J. Low Temp. Phys. 122, 527 (2001).

14. N. Schwentner, E.E. Koch, and J. Jortner, Electronic Excitation in Condensed Rare Gases, Springer Tracts in Modern Physics, Vol. 107, Springer, Berlin (1985).

15. D.S. Tinti and G.W. Robinson, J. Chem. Phys. 49, 3229 (1968)

16. R.J. Sayer, R.H. Prince, and W.W. Duley, Phys. Status Solidi B106, 249 (1981).
17. E.V. Savchenko, O.N. Grigorashchenko, G.B. Gumenchuk, A.N. Ogurtsov, M. Frankowski, A.M. SmithGicklhorn, and V.E. Bondybey, Radiat. Eff. Def. Solids 157, 729 (2002).

18. Yu.I. Rybalko, E.V. Savchenko, and I.Ya. Fugol', Fiz. Nizk. Temp. 11, 637 (1985) [Sov. J. Low Temp. Phys. 11, 349 (1985)].

19. E. Schuberth and M. Creuzburg, Phys. Status Solidi B71, 797 (1975)

20. Ch.B. Lushchik, JETP 3, 390 (1956). 